\title{
Distribution and molecular analysis of Subtilase cytotoxin gene (subAB) variants in Shiga toxin-producing Escherichia coli (STEC) isolated from different sources in Iran
}

\author{
Mahdi Askari Badouei ${ }^{1}$, Maziar Jajarmi ${ }^{2}$, Aria Narimani ${ }^{3}$, Taghi Zahraei Salehi ${ }^{4}$, Reza \\ Ghanbarpour $^{5}$, Stefano Morabito ${ }^{6}$, and Alireza Koochakzadeh ${ }^{7}$ \\ ${ }^{1}$ Ferdowsi University of Mashhad Faculty of Veterinary Medicine \\ ${ }^{2}$ Shahid Bahonar University Faculty of Veterinary Medicine \\ ${ }^{3}$ Islamic Azad University Garmsar Branch \\ ${ }^{4}$ University of Tehran, Faculty of Veterinary Medicine \\ ${ }^{5}$ Faculty of Veterinary Medicine, Shahid Bahonar University of Kerman \\ ${ }^{6}$ European Union Reference Laboratory for Escherichia coli, Istituto Superiore di Sanita, \\ Dipartimento di Sicurezza Alimentare, Nutrizione e Sanita Pubblica Veterinaria, Rome, \\ Italy \\ ${ }^{7}$ University of Tehran Faculty of Veterinary Medicine
}

November 19, 2020

\begin{abstract}
Subtilase is a potent cytotoxin that was first described in O113:H21 strain in Australia as a plasmid- encoded cytotoxin (subAB1). Subsequently, chromosomal variants including subAB2-1, subAB2-2, and subAB2-3 were described. In the present study a collection of 101 STECs isolated from various sources in Iran (2009-2016) were analyzed for the detection of different genes encoding the subtilase variants, plasmidic and chromosomal virulence genes, together with the phylogroup and serogroups. Overall, 57 isolates (56.4\%) carried at least one variant of subAB. Most strains from small ruminants including $93 \%$ of sheep and $96 \%$ of caprine isolates carried at least one chromosomally encoded variant (subAB-2). In contrast, 12 cattle isolates (24\%) only harbored the plasmid encoded variant (subAB1). STEC strains from other sources including deer, pony and humans were positive for subAB-2-1 and/or subAb2-2. Concerning the virulence markers, some strains showed an association with hosts the bacteria were isolated from. In particular, tia was associated with sheep, goats and pony isolates and astA gene was present in deer, pony and goats and terD was only found in deer and pony isolates. Only cattle STEC carried espP and epeA, the important markers of pO113 plasmid. Some genes were widespread among strain of various sources like ehly, iha and lpfO113 and some genes were not detected such as efa1, toxB and katP. Most strains belonged to phylogenetic group B1 (89.47\%), but five strains from cattle, deer, pony and a goat were assigned to A phylogroup. Most cattle strains belonged to O113, while O5 was just detected in ovine isolates, and O128 and O113 were present in caprine strains. In conclusion, the present study reveals the presence of potentially pathogenic genotypes among LEE-negative isolates and some host specificity related to subtilase variants and other virulence markers that may aid in source tracking of STEC during outbreaks.
\end{abstract}

\section{Hosted file}

Subtilase final- Transbundary.pdf available at https://authorea.com/users/377125/articles/ 493914-distribution-and-molecular-analysis-of-subtilase-cytotoxin-gene-subab-variantsin-shiga-toxin-producing-escherichia-coli-stec-isolated-from-different-sources-in-iran

\section{Hosted file}


Table 1.pdf available at https://authorea.com/users/377125/articles/493914-distribution-andmolecular-analysis-of-subtilase-cytotoxin-gene-subab-variants-in-shiga-toxin-producingescherichia-coli-stec-isolated-from-different-sources-in-iran 